\title{
TITLE:
}

\section{$<$ Note>Biological and molecular characterization of Citrus tatter leaf virus in Taiwan}

\author{
$\operatorname{AUTHOR}(\mathrm{S}):$ \\ Lin, Chun-Yi
}

\section{CITATION:}

Lin, Chun-Yi. <Note>Biological and molecular characterization of Citrus tatter leaf virus in Taiwan. Sustainable humanosphere : bulletin of Research Institute for Sustainable

Humanosphere Kyoto University 2019, 15: 1-4

ISSUE DATE:

2019-09-30

URL:

http://hdl.handle.net/2433/245068

RIGHT: 


\section{Biological and molecular characterization of Citrus tatter leaf virus in Taiwan}

\section{(Center for Exploratory Research on Humanosphere, RISH, Kyoto University)}

\section{Chun-Yi Lin}

Citrus tatter leaf virus (CTLV) causes chlorotic leaves with bud union incompatibility in citrus scion and its current incidence in Taiwan is $65 \%$. However, the burden of infection has long been underestimated due to its common latency and the serious threat of tatter leaf disease should be investigated and addressed without delay. Here, we evaluated the biological characteristics and genome sequences of Taiwanese isolates. Three full-length sequenced local isolates and phylogenetic analyses revealed similarities with isolates from neighbouring countries, indicating the possible origins of local isolates. Two mild isolates obtained from Liuchen sweet orange (LCd-NA-1) and Kumquat (Kq-6-2-46) were inoculated on indicator plants and exhibited differences in foliar symptom expression and temperature tolerance compared with foreign isolates causing severe disease. In summary, the study provided comprehensive information and a foundation for citrus virus management or further research.

\section{Introduction}

Citrus tatter leaf was first reported in latently infected Meyer lemon trees (Citrus limon L. Burm.f.) in California imported from China [1]. Typical local chlorotic lesions and systemic mosaic symptoms on the leaves of herbaceous plants have been observed following mechanical CTLV inoculation $[2,3,4]$. CTLV inoculation also causes tattered and chlorotic leaves with bud union incompatibility in citrus scion grafted on Citrus excelsa, Rusk trifoliate and Troyer citranges or swingle citrumelos [5]. CTLV is primarily transmitted via mechanical inoculation and infected bud propagation, but no insect vectors have been identified [6].

CTLV is a member of the genus Capillovirus (family Betaflexiviridae), which includes two species, Cherry virus A (CVA) and Apple stem grooving virus (ASGV) [7]. The single-stranded, positive-sense genomic CTLV RNA is 6496 nt in length with a 5' cap and 3' poly adenine tail. This sequence contains two open reading frames (ORF1 and ORF2), and multiple functional proteins are translated to perform biological functions.

Tatter leaf disease, one of the four major systemic and endemic citrus diseases in Taiwan, has long been present and is commonly detected up to $50 \%$ of total citrus plants in field surveys [8]. Because of its latent infection characteristics, the severity of tatter leaf disease has been underestimated, and few CTLV-related studies have been conducted worldwide. Here, we determined the biological characteristics of different isolates from Rusk citrange or C. quinoa. This study provides comprehensive and basic information for CTLV management and further research.

\section{Symptom expression as determined in the CTLV bioassay}

In the Rusk citrange test, the indicator plants were more susceptible to Chinese isolates (Table 1). The Sat-HY-2 (Chinese) isolate caused severe leaf curling and chlorosis, whereas the Cal-KS-1 isolate induced severe tatter and distorted leaves 4-8 weeks after inoculation. In contrast, the LCd-NA-1 (Taiwanese) isolate caused mild symptoms, including slight leaf curl, while the Kq-6-2-46 isolate caused severe, obviously chlorotic spots accompanied by leaf distortion (Fig. 1a). In the C. quinoa test, the indicator plants were more sensitive to Chinese isolates at two temperatures (Table 1). Under high temperature conditions, symptoms developed more rapidly (5-10 days) following infection with the two Chinese isolates than with the Taiwanese isolate (6-12 days). The Chinese isolates both caused obvious local chlorotic lesions with clear edges on inoculated leaves; the Cal-KS-1 isolate also induced heightened mosaic, chlorotic, curling and epinasty symptoms on systemic top leaves compared with Sat-HY-2 infection. The Kq-6-2-46 isolate caused mild local lesions on inoculated leaves, whereas few chlorotic spots were observed on LCd-NA-1-infected leaves. Moreover, only LCd-NA-1 induced mosaic chlorotic spots on systemic top leaves (Fig. 1b). Symptom development is slowed under low temperature conditions (20-22 days). 


\section{NOTE}

Table 1. Comparison of symptom expression on Rusk citrange and Chenopodium quinoa infected by four citrus tatter leaf virus (CTLV) isolates.

\begin{tabular}{|c|c|c|c|c|c|c|c|c|}
\hline \multirow[b]{2}{*}{ Indicator plant } & \multicolumn{8}{|l|}{ CTLV isolate ${ }^{a}$} \\
\hline & Sat-HY-2 (C) & & Cal-KS-1 (C) & & LCd-NA-1 (T) & & $\mathrm{Kq}-6-2-46(\mathrm{~T})$ & \\
\hline Rusk citrange (leaf) & $+^{b}$ & & + & & ++ & & + & \\
\hline Chlorotic spots & 3 & & 2 & & 1 & & 3 & \\
\hline Leaf distortion & 3 & & 2 & & 1 & & 0 & \\
\hline Leaf tattering & 3 & & 3 & & 0 & & 1 & \\
\hline C. quinaa & $30{ }^{\circ} \mathrm{C}$ & $22^{\circ} \mathrm{C}$ & $30^{\circ} \mathrm{C}$ & $22{ }^{\circ} \mathrm{C}$ & $30{ }^{\circ} \mathrm{C}$ & $22{ }^{\circ} \mathrm{C}$ & $30^{\circ} \mathrm{C}$ & $22{ }^{\circ} \mathrm{C}$ \\
\hline Inoculated leaves & + & + & + & + & + & +H & + & + \\
\hline Local lesions & 3 & 3 & 3 & 3 & 1 & 1 & 2 & 1 \\
\hline Uninoculated leaves & - & + & + & + & + & + & + & + \\
\hline Mottling & 0 & 3 & 3 & 0 & 1 & 2 & 0 & 2 \\
\hline Epinasty & 0 & 3 & 3 & 0 & 0 & 2 & 0 & 2 \\
\hline Leaf curling & 0 & 3 & 3 & 0 & 0 & 2 & 0 & 2 \\
\hline
\end{tabular}

Symptom index: 0 , symptomless; 1 , mild; 2 , moderate; 3 , severe. Symptom expression on Rusk citrange was recorded 3 months after inoculation by bud grafting.

a(C), Chinese isolate; (T) Taiwanese isolate.

${ }^{6}$ RT-PCR detection of CTLV. Pixel value index of the CTLV-specific band on agarose gel measured by a densitometer:,$-<30 ;+, 30-79 ;++$, 80-129; +++, 130-180.

Obvious partially chlorotic spots were observed on both Chinese isolate-infected leaves, while larger and expanded spots were observed on Sat-HY-2 infected leaves. The opposite results were shown in systemic symptoms. Severely mosaic, chlorotic leaves with curling and distortion were observed on the Sat-HY-2 infected plants, whereas no systemic symptoms were observed with the Cal-KS-1 infection. The two Taiwanese isolates caused no obvious symptoms on inoculated leaves but did cause distortion and curling symptoms on systemically infected leaves (Fig. 1b).

Figure 1. Comparison of foliar symptoms on Rusk citrange (a) and C. quinoa (b) following infection with four distinct CTLV isolates. Symptom expression on Rusk citrange and $C$. quinoa was observed at six weeks and one week after inoculation, respectively. CTLV inoculation tests on $C$. quinoa were conducted in greenhouses at $22^{\circ} \mathrm{C}$ and $30^{\circ} \mathrm{C}$. Sat, Satsuma mandarin; Cal, Calamondin; LCd, Liuchen sweet orange; Kq, Kumquat.

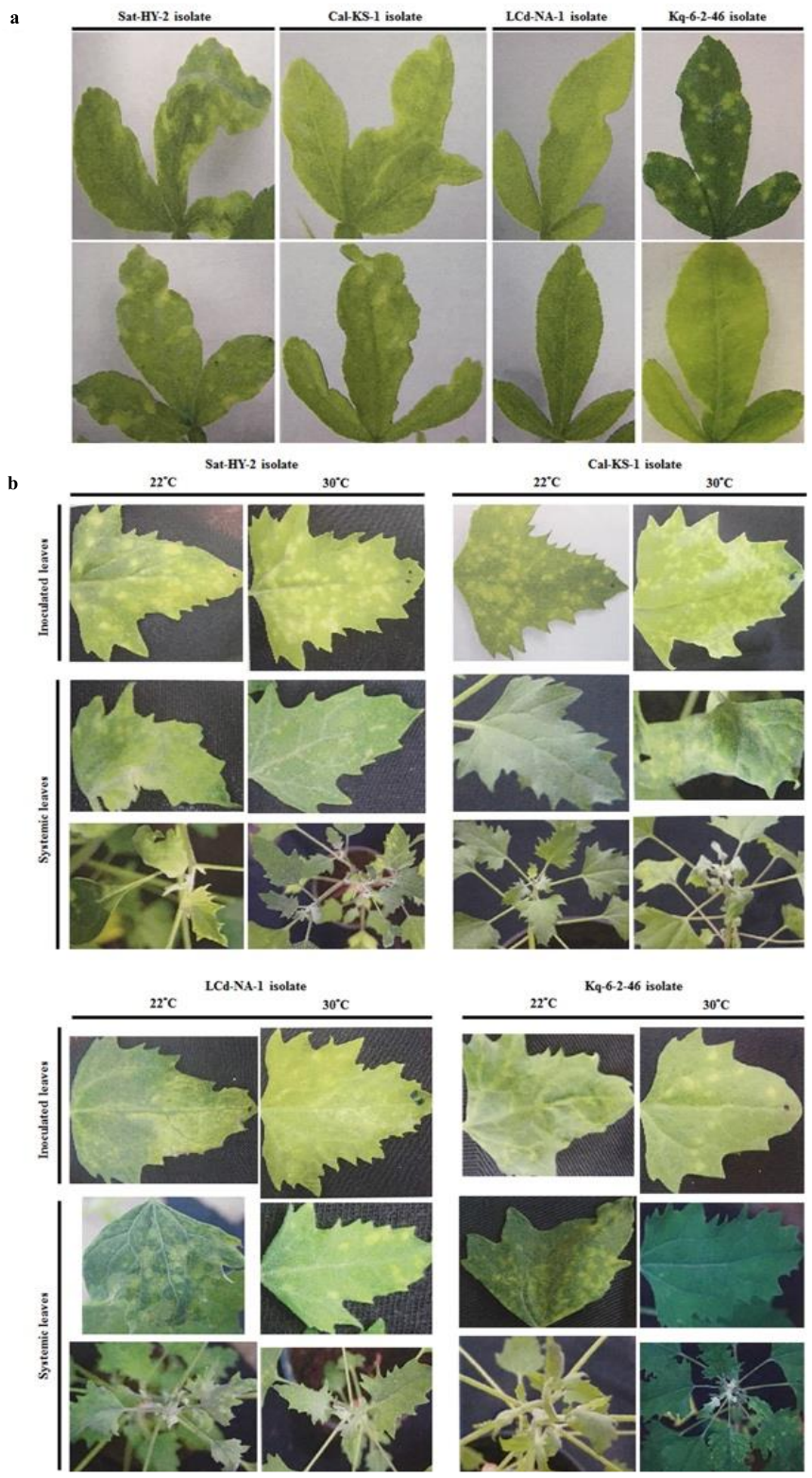




\section{NOTE}

\section{CTLV distribution in different citrus tissues}

RT-PCR detection was performed on different tissues from two CTLV-infected citrus cultivars (Kumquat and Minneola tangelo). Strong CTLV signals were detected in mature and young leaves and stem bark, whereas moderate signals were present in the root, midrib and fruit. No signals were detected in flower tissues (Fig. 2).

Figure 2. Analysis of CTLV distribution in different citrus tissues by RT-PCR. (a) CTLV-infected Minneola tangelo cultivated in the citrus nursery of Chiayi Agriculture Experiment Station (CAES). (b) RT-PCR assay for CTLV detection in different tissues of host citrus plants. The product size was 527 bp. Lane 1, root; 2, mature leaf; 3, young leaf; 4, midribs; 5, bark; 6, flower; 7, fruit transportation tissue. M, 100-bp DNA ladder marker. a

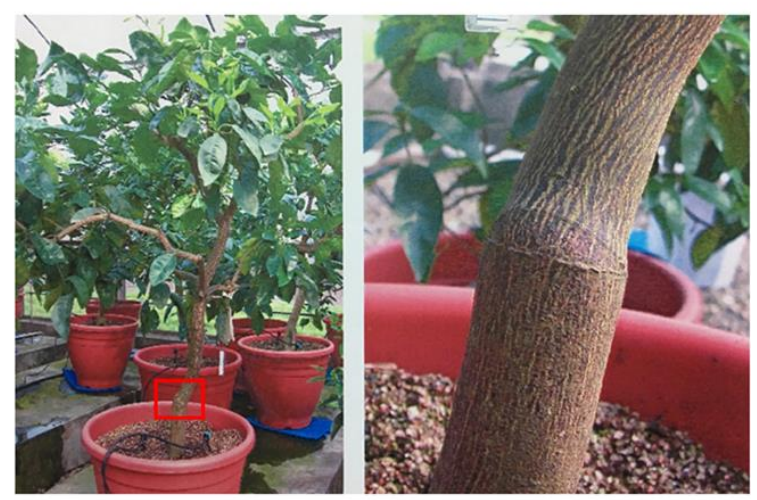

b

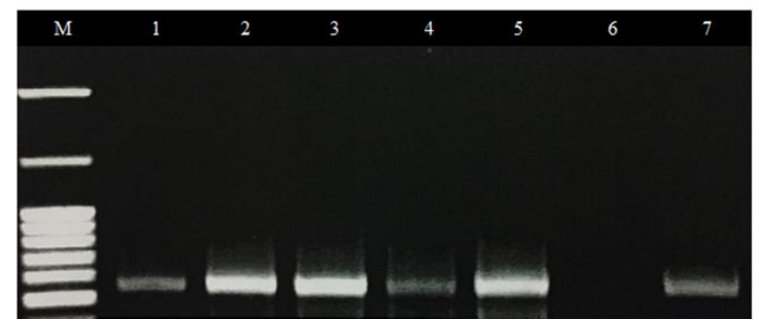

Genomic, amino acid and phylogenetic analyses a of CTLV

As shown in Table 2, 79.4-94\% similarity was observed for nucleotide alignment when compared to CTLV-Pk. Amino acid (aa) alignment demonstrated high similarities for the ORF1 (85.395.8\%), CP (92-95.8\%) and MP (93.4-99.1\%) sequences compared to CTLV-Pk. Obvious variations in variable regions I (14.3-18.2\%) and II (56.3-63.6\%) exist among the isolates from Taiwan and other countries (Table 2). Based on phylogenetic analyses of the ORF1 genome sequences (Fig. 3a), the Taiwanese isolates were similar to isolates from the USA (CTLV-ML) and China (CTLV-SO). In addition, phylogenetic analysis based on aa sequences indicated the Taiwanese isolates were similar to the Chinese isolates which comes from the same group, whereas the Japanese isolates belong to another group (Fig. $3 b)$.

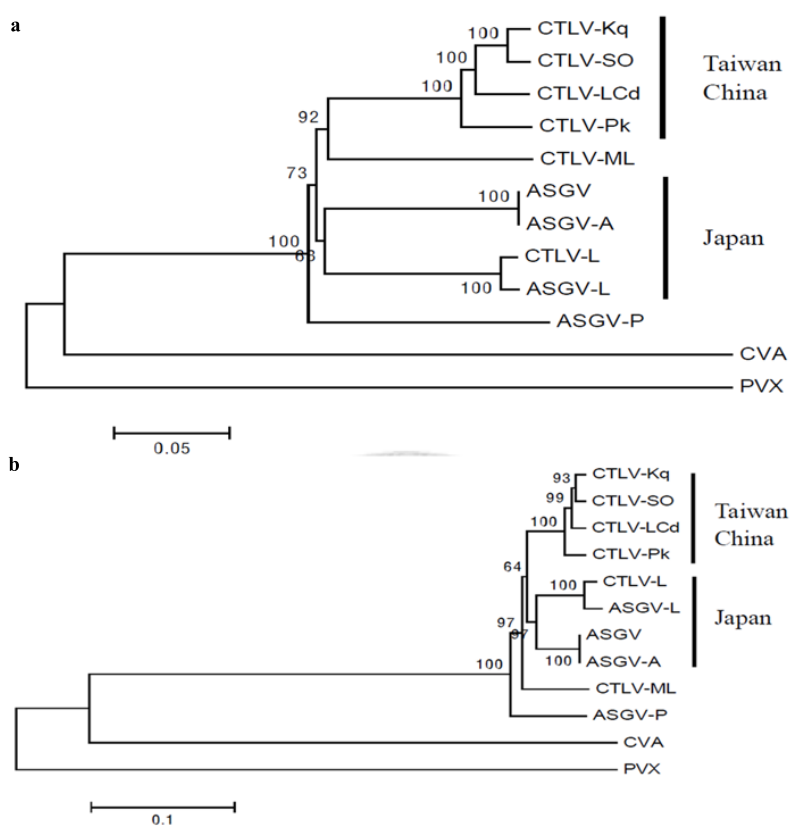

Figure 3. Phylogenetic trees derived from full-length genome sequences (a) and ORF1 aa sequences (b) of citrus tatter leaf virus (CTLV) isolates from Ponkan mandarin (CTLV-Pk) and other capilloviruses. Trees were constructed with MEGA 5.0 using the UPGMA method with 1000 bootstrap replications. Potato virus X (PVX) (JF430080) was used as the out-group. Bars indicate numbers of nucleotide substitutions per site. 


\section{NOTE}

Table 2. Comparison of genome sequences and amino acid (aa) identities (\%) of open reading frame (ORF1) polyprotein, coat protein (CP), movement protein (MP) and variable regions (VRI and VRII) among Ponkan isolate of citrus tatter leaf virus (CTLV-Pk) and other capilloviruses.

\begin{tabular}{llllllll}
\hline Sequence & CTLV-Pk & CTLV-Kq & CTLV-LCd & CTLV-ML & CTLV-L & ASGV & ASGV-P \\
\hline Genome & 100 & 94.0 & 93.9 & 81.9 & 81.7 & 81.7 & 79.4 \\
ORF1 aa & 100 & 95.0 & 95.8 & 86.9 & 86.9 & 87.6 & 85.3 \\
CP aa & 100 & 95.4 & 95.8 & 92.0 & 94.5 & 93.7 & 92.0 \\
MP aa & 100 & 99.1 & 98.1 & 95.9 & 96.9 & 95.6 & 93.4 \\
VRI aa & 100 & 70.0 & 80.0 & 14.3 & 23.8 & 14.3 & 18.2 \\
VRII aa & 100 & 85.0 & 87.4 & 63.6 & 56.3 & 57.0 & nd \\
\hline
\end{tabular}

CTLV from Ponkan mandarin (CTLV-Pk), kumquat (CTLV-Kq), Liuchen sweet orange (CTLV-LCd), Meyer lemon (CTLV-ML), lily (CTLV-L); apple stem grooving virus from apple (ASGV), pear black necrotic leaf spot isolate of ASGV (ASGV-P); cherry virus A (CVA).

nd, not determined.

\section{Conclusion}

In this study, we focused on the biological and molecular characterisation of CTLV isolates and improved the sensitivity and specificity of available detection methods. Standard protocols, including a bioassay and molecular detection, are essential tools to prevent infection by exotic and frequently severe isolates, and permit the early detection and removal of local latent infections. In the future, the construction of an infectious CTLV clone could represents a useful tool for further functional and genetic research on virus replication, movement and pathogenicity. The artificial clone will also benefit the development of a virus-induced gene silencing (VIGS) vector for further in planta studies. The study provides basic biological results which may help industry to select tolerant cultivars to prevent viral infection and construct suitable environmental factors to growth virus-free citrus seedlings. Uneven distributions of CTLV in different citrus tissues could give important suggestion to establish standard operation procedure in quarantine protocol. The study provides valuable aspects for citrus management strategy to citrus industry and government people.

\section{Acknowledgements}

We thank the kind supply of several citrus cultivars and samples by the farmers of Taiwan to complete the field survey. The authors also thank Prof. Hong-Ji, Su from National Taiwan University for collecting foreign isolates and giving advices on the manuscript.

\section{References}

[1] Wallace, J. M., and R. J. Drake, "Tatter-leaf, a previously undescribed virus effect on citrus", Plant Disease Reporter, vol. 46, 211-212, 1962.

[2] Yarwood, C. E., "Mechanical transmission of a latent lemon virus", Phytopathology, vol. 53, pp. 1145, 1963.

[3] Fulton, R. W., "Mechanical transmission of tatter leaf virus from cowpea to citrus", Phytopathology, vol. 56, pp. 575-576, 1966.

[4] Garnsey, S.M., "Mechanical transmission of a virus that produces tatter leaf symptoms in Citrus excelsa", In: Weathers LG, M Cohen, eds. Proceeding of the $6^{\text {th }}$ Conference of the International Organization of Citrus Virologist. IOCV: University of California Riverside, USA, pp. 137, 1974.

[5] Zhang, T.M., X. Y. Liang, and C. N. Roistacher, "Occurrence and detection of citrus tatter leaf virus (CTLV) in Huangyan, Zhejiang Province, China", Plant Disease, vol. 72, pp. 543-545, 1988.

[6] Miyakawa, T., and C. Matsui, "A bud-union abnormality of Satsuma mandarin on Poncirus trifoliata rootstock in Japan", In: Calavan EC, ed. Proceeding of the $7^{\text {th }}$ Conference of the International Organization of Citrus Virologist. IOCV: University of California Riverside, USA, pp. 125-131, 1976.

[7] Martelli, G.P., M. J. Adams, J. F. Kreuze, V. V. Dolja, "Family Flexiviridae: A case study in virion and genome plasticity", Annual Review of Phytopathology, vol. 45, pp. 73-100, 2007.

[8] Tsai, C.H., H. J. Su, Y. C. Feng, and T. H. Hung, "Study of citrus Huanglongbing and its complex infection with Citrus tristeza closterovirus and Citrus tatter leaf capillovirus in Taiwan", Plant Pathology Bulletin, vol. 16, pp. 121-129, 2007. 